\title{
Erratum
}

\section{Erratum to: Targeting the testis-specific heat-shock protein 70-2 (HSP70-2) reduces cellular growth, migration, and invasion in renal cell carcinoma cells}

Swarnendra Singh and Anil Suri

[Tumor Biology, 35, 2014, 12695-12706, DOI: 10.1007/s13277-014-2594-5]

In the published version of Figure 4, 4B migration ability of CaKi-1 cells with HSP70-2 shRNA T4 treatment was inadvertently presented with the same image of Figure 5, 5B invasion ability of CaKi-1 cells with HSP70-2 shRNA $\mathrm{T}_{\mathrm{S}}$ treatment during figure assembly for manuscript submission. We have accordingly replaced HSP70-2 shRNA $\mathrm{T}_{\mathrm{S}}$ treatment image in corrected Figure 5, 5B.

Figure 5, 5A invasion ability of A704 cells with HSP70-2 shRNA $\mathrm{T}_{\mathrm{S}}$ treatment was inadvertently presented with the overlapping field images of HSP70-2 shRNA T4 treatment during figure assembly for manuscript submission. We have accordingly replaced HSP70-2 shRNA $T_{S}$ treatment image in Corrected Figure 5, 5A.

Figure 5, 5B invasion ability of CaKi-1 cells, only cells image was inadvertently presented with the overlapping field images with CaKi-1 cells HSP70-2 shRNA $\mathrm{T}_{4}$ treatment during figure assembly for manuscript submission. We have accordingly replaced only cells image in corrected Figure 5, 5B.

It was a copy and paste error during image processing. However we have accordingly corrected and replaced the Figure 5A and Figure 5B.

Change in representative image does not affect the interpretation in Figure 5. The corrected Figure 5 is enclosed. The figure legend is correct as published and is also shown for reference below. The data were not manipulated in anyway, the error has no bearings on the interpretation of the results, nor they influence the conclusions of the work. 
A A704

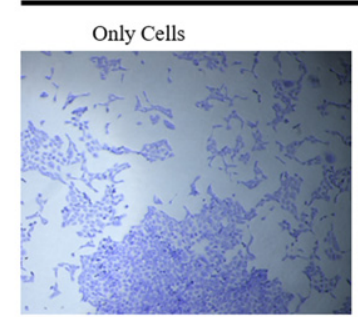

HSP70-2 ShRNA $\mathrm{T}_{3}$
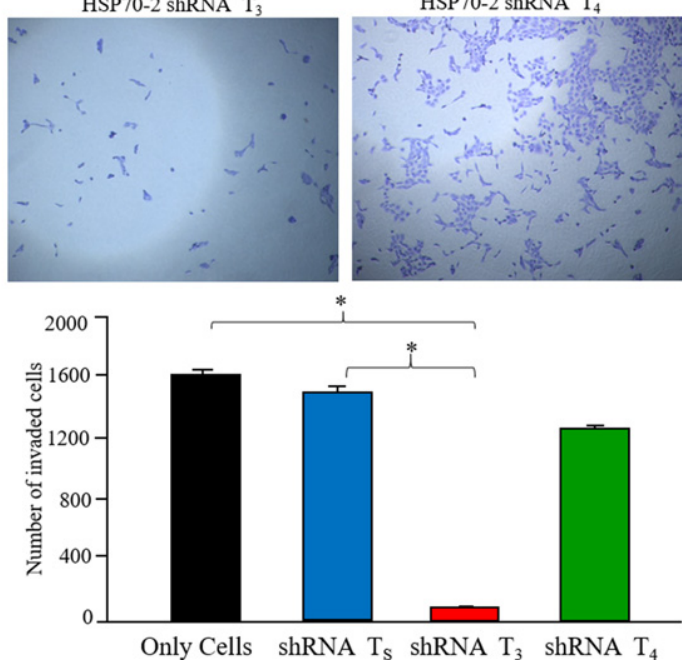

B

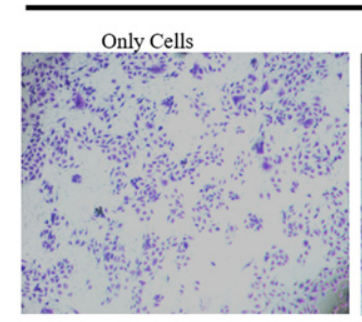

HSP70-2 ShRNA $T_{3}$
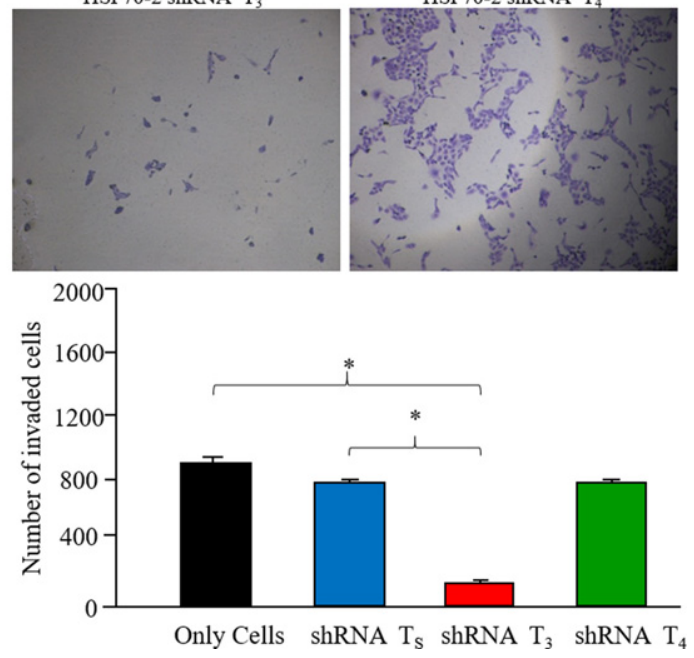

Fig. 5. HSP70-2 knockdown inhibits the invasive property of RCC cells. Invasion assay were carried out in A704 and Caki-1 cells by transient transfection using HSP70-2 shRNA $\mathrm{T}_{3}$ or shRNA $\mathrm{T}_{4}$ or shRNA $\mathrm{T}_{\mathrm{S}}$. (a) A704 and (b) Caki-1 cells showing reduction in number of invaded cells when transfected with HSP70-2 shRNA $T_{3}$ as compared to the cells transfected with shRNA $T_{4}$ or shRNA $T_{S}$. However only cells failed to show any significant inhibition in invasive property of cancer cells. Representative histogram revealed significant reduction in number of invaded cells when transfected with shRNA $\mathrm{T}_{3}$ as compared to cells transfected with shRNA $\mathrm{T}_{\mathrm{S}}$ or shRNA $\mathrm{T}_{4}$ or only cells. $n=3$ independent experiments; each experiment was performed in triplicate. Points, mean; bars, SE. statistical significance *, $P<0.0001$. 